\title{
HEIME
}

\section{"Wir sollen Akten pflegen, nicht die Menschen«}

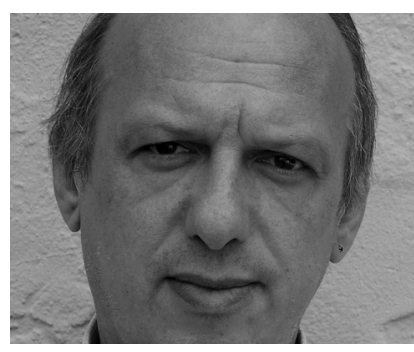

VON MICHAEL GRABER-DÜNOW Michael Graber-Dünow ist Sozialarbeiter und Altenpfleger. Nach mehrjähriger Tätigkeit in beiden Berufen wechselte er 1988 in das Leitungsteam eines großen Frankfurter Altenhilfezentrums. Seit 1997 ist er Leiter des Altenpflegeheims Justina von Cronstetten Stift in Frankfurt am Main. Er ist außerdem im Fortbildungsbereich sowie als Fachbuchautor tätig. E-Mail altenpflegeheim@justinavon-cronstetten-stift.de

\begin{abstract}
Rund 980 bundesweit geltende Vorschriften müssen Heime in Deutschland beachten. Die Bürokratie in Altenpflegeheimen scheint seit dieser Zählung vor sieben Jahren nicht geringer worden zu sein, wie pflegebedürftige Menschen und Praktiker monieren.
\end{abstract}

Das Problem der zunehmenden Bürokratisierung der Altenpflege ist schon seit Jahren bekannt. Vor allem die immer weiter ausufernden Anforderungen an die Verschriftlichung aller Pflege- und Betreuungsmaßnahmen stehen im Fokus der Kritik vieler Praktiker.

Dabei dürfte zwar unbestritten sein, dass eine Pflegedokumentation und Pflegeprozessplanung dem derzeitigen Stand der pflegewissenschaftlichen Erkenntnisse entspricht. Der prinzipielle Widerspruch besteht allerdings darin, dass diese Anforderungen in Elfenbeintürmen entwickelt wurden, ohne die real existierenden personellen Ressourcen in den Heimen zu berücksichtigen. Nach den Vorgaben der Kostenträger bewegen sich diese Ressourcen nämlich auf einem so niedrigen Niveau, dass eine ganzheitliche, bedürfnisorientierte Pflege und Betreuung der Bewohner selbst bei größtem Engagement der Mitarbeiterinnen und Mitarbeiter schon vor der sukzessiven Einführung einer geregelten Dokumentation kaum zu realisieren war.

Die Heime stehen somit vor der unlösbaren Aufgabe mit einer seit Einführung der Pflegeversicherung sogar tendenziell rückläufigen Personalausstattung immer weiter ansteigenden bürokratischen Anforderungen gerecht werden zu müssen. Ein Spagat, der nicht gelingen kann, ohne entweder die Dokumentationspflichten oder die Versorgung der Bewohner zu vernachlässigen. Wie der Verband Deutscher Alten- und Behindertenhilfe bereits im Jahre 2003 feststellte, blieben den Pflegekräften schon damals nur noch 62 Prozent ihrer Arbeitszeit für praktische Pflegetätigkeiten. Auch wenn dazu keine aktuellen Zahlen vorliegen, ist anzunehmen, dass sich dieser Wert seither noch weiter verringert hat.

Ein weiteres bürokratisches Problem besteht in der Überregulierung der Heime durch die unkoordinierten und teilweise wi- dersprüchlichen Kontrollen verschiedener externer Aufsichtsbehörden. So zählte ebenfalls im Jahre 2003 die Forschungsgesellschaft für Gerontologie mehr als 980 bundesweit geltende Vorschriften, die Heime zu beachten haben.

Aufgrund der skizzierten Zusammenhänge erarbeitete der von der damaligen Bundesregierung initiierte »Runde Tisch Pflege « Vorschläge zur Entbürokratisierung der Altenpflege, die 2005 veröffentlicht wurden. Fünf Jahre später ist jedoch festzuhalten, dass dies nicht nur ohne praktische Folgen blieb, sondern sich die Situation seither sogar noch weiter verschärfte.

In den verbalen öffentlichen Bekundungen von Politikern sind »Bürokratieabbau " und "Verbesserung der Pflegequalität « zwar noch immer beliebte Schlagworte, die auf die Praxis allerdings keinerlei Auswirkungen haben.

Ganz im Gegenteil: Ein weiterer Ausbau der bürokratischen Anforderungen führt bei gleichbleibenden personellen und somit zeitlichen Ressourcen letztlich zu einer Verschlechterung der Pflegequalität, da den Mitarbeitenden in den Heimen immer weniger Zeit für die Befriedigung von Bewohnerbedürfnissen verbleibt.

\section{Kontrollen harmonisieren}

Externe Prüfungen von Pflegeeinrichtungen sind zweifellos unverzichtbar. Die Kontrollen werden jedoch noch immer von einer Vielzahl von Aufsichtsbehörden durchgeführt, so dass Doppel- oder sogar Dreifachprüfungen derselben Sachverhalte - teilweise zudem mit unterschiedlichen Ergebnissen - an der Tagesordnung sind. Mit Einführung des Pflege-Weiterentwicklungsgesetzes vom 1. Juli 2008 ist eine weitere Regelprüfung hinzugekommen: Bis Ende 2010 müssen alle Pflegeeinrichtungen vom Medizinischen Dienst der Krankenkassen (MDK) einmal überprüft werden. Ab dem Jahr 2011 hat die Prüfung dann jährlich zu erfolgen, so dass der auch schon zuvor bestehende Prüfauftrag des MDK nun in einer hohen Frequenz regelhaft festgeschrieben ist. Diese Kontrollen sind gemäß \117 SGB XI zwar mit den Überprüfungen nach dem Heimgesetz zu harmo- 
nisieren, doch davon ist in der Praxis nichts festzustellen.

Der MDK-Prüfkatalog wurde nach dem Willen des Gesetzgebers gemeinsam mit den Leistungserbringern erarbeitet. Angesichts der im Folgenden beschriebenen Konsequenzen dieser Prüfungen stellt sich allerdings die Frage, ob die Vertreter der
Pflegeeinrichtungen ihren Aufgaben in den Gremien gerecht geworden sind.

\section{Transparenz durch Schulnoten?}

Der Kontrollauftrag des MDK ist in vier Bereiche mit einer unterschiedlichen Anzahl von Prüfkriterien gegliedert:
- Pflege und medizinische Versorgung (35 Kriterien)

- Umgang mit demenzkranken Bewohnern (10 Kriterien)

n soziale Betreuung und Alltagsgestaltung (10 Kriterien)

- Wohnen, Verpflegung, Hauswirtschaft und Hygiene (9 Kriterien)

\section{Was ist eigentlich der MDK?}

Der Medizinischer Dienst der Krankenversicherung (MDK) ist der medizinische Begutachtungs- und Beratungsdienst der gesetzlichen Krankenkassen und Pflegekassen. Er nimmt vielfältige Aufgaben für seine Träger wahr, u. a. bei Pflegebedürftigkeit und Rehabilitation. [...]

Die Rechtsgrundlagen des MDK finden sich im Neunten Kapitel des Sozialgesetzbuches (SGB) V ( $\$ \$ 275-283)$. Der MDK ist in der Regel landesweit organisiert und wird von den Landesverbänden der Krankenkassen und den Vertretungen der Ersatzkassen im Land getragen. Er ist eine eigenständige Arbeitsgemeinschaft, die überwiegend mit eigenem, beim MDK angestellten Personal arbeitet. Kerngruppen der gutachterlich Tätigen sind Ärzte, die in allen Feldern begutachtend und beratend tätig sind und Pflegefachkräfte, die überwiegend in der Pflegebegutachtung und Qualitätsprüfung von Pflegeeinrichtungen tätig sind.

Grundlage der Tätigkeit der Gutachter des MDK ist das Prinzip der fachlichen Unabhängigkeit ( $\$ 275$ Abs. 5 SGB V). Bei der Beurteilung medizinischer und pflegerischer Sachverhalte unterliegen die Gutachter des MDK keinen Weisungen der Auftraggeber.

Die Finanzierung des MDK erfolgt durch eine Umlage, die für jedes Mitglied in der gesetzlichen Kranken- und Pflegeversicherung erhoben wird. Damit sind die Aufträge an den MDK durch die Gemeinschaft aller Krankenkassen und damit unabhängig von der konkreten Inanspruchnahme finanziert. Nutzerbezogene Entgelte werden bei Aufträgen Dritter, z. B. der Sozialämter erhoben. Auf Bundesebene nimmt der Medizinische Dienst der Spitzenverbände der Krankenkassen (MDS) die Aufgaben einer Spitzenorganisation wahr. Seine Aufgabe ist v. a. die Koordinierung der Arbeit der MDK. [...]

\section{Aufgaben für die Pflegeversicherung:}

1. Begutachtung bei Pflegebedürftigkeit. Das SGB XI schreibt vor, dass die Pflegekassen den MDK mit den Ermittlungen zur Feststellung der Pflegebedürftigkeit zu beauftragen haben. Dies gilt sowohl für die häusliche als auch für die stationäre Pflege. Die Ermittlungen umfassen sowohl die Untersuchung der medizinischen Voraussetzungen der Pflegebedürftigkeit, die Ermittlung des Hilfebedarfs anhand der Verrichtungen des täglichen Lebens als auch die Empfehlung zu Pflegestufen. Der individuelle Hilfebedarf des Pflegebedürftigen entscheidet über die Einstufung in eine der drei Pflegestufen. [...]

2. Qualitätsprüfung von Pflegeeinrichtungen. Neben den Begutachtungsaufgaben obliegt dem MDK auch die Prüfung der Qualität von Pflegediensten und Pflegeheimen. Dabei wird der MDK im Auftrag der Landesverbände der Pflegekas- sen tätig. Die Qualitätsprüfungen des MDK umfassen die Prüfung der Struktur-, Prozess- und Ergebnisqualität der Pflege. Der erstellte Prüfbericht wird der Einrichtung, den Pflegekassen und der Heimaufsicht zur Verfügung gestellt. Der MDK vertritt eine beratungsorientierte Prüfkonzeption, $d . h$. die Prüfberichte dienen in erster Linie dazu, der Einrichtung den Stand ihrer Qualitätsentwicklung zu spiegeln und ihr damit Anregungen für qualitätsverbessernde Prozesse zu geben. Bei erheblichen Qualitätsdefiziten können die Pflegekassen der Pflegeeinrichtung Auflagen erteilen oder bei extremen Defiziten sogar den Versorgungsvertrag kündigen.

\section{Aufgaben für die Krankenversicherung:}

1. Einzelfallbezogene Begutachtung. Die Krankenkassen können in allen Leistungsangelegenheiten eine gutachtliche Stellungnahme des MDK einholen. Ziel ist es dabei, die medizinischen Voraussetzungen der Notwendigkeit, Zweckmäßigkeit und Wirtschaftlichkeit sowie die Qualität der Leistungen zu prüfen. Wesentliche Begutachtungsbereiche sind Krankenhaus- und Rehabilitationsleistungen, die Versorgung mit Hilfsmitteln, die zahnmedizinische Versorgung, die Prüfung von Arbeitsunfähigkeit sowie unkonventionellen Heilmethoden. [...]

2. Strukturprüfung. Neben den Einzelfallprüfungen haben sich inzwischen auch Strukturprüfungen von Einrichtungen etabliert. Kennzeichen der Strukturprüfungen ist die Einschätzung der Gesamtversorgungssituation einer Einrichtung über die Prüfung einer relevanten Zahl von Einzelfällen durch Stichproben. Eine Stichprobenprüfung ist insbesondere nach $\$ 17$ C KHG im Krankenhausbereich vorgesehen. Konkret werden hier die Notwendigkeit der stationären Aufnahme und die korrekte Kodierung der Abrechnungsfälle im Krankenhaus geprüft. [...]

3. Grundsatzberatung. Der MDK und der MDS beraten die Krankenkassen und deren Verbände in allen allgemein medizinischen und pflegerischen Fragen. Ein wesentliches Arbeitsfeld ist die Bewertung neuer und bestehender medizinischer Verfahren. Weitere wichtige Beratungsfelder sind die Weiterentwicklung von Versorgungsstrukturen und Vergütungen. $[\ldots]$

Peter Pick

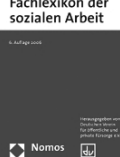

Quelle: Deutscher Verein für öffentliche und private Fürsorge e. V. (Hg.): Fachlexikon der sozialen Arbeit. 6. Auflage. Nomos Verlagsgesellschaft, Baden-Baden 2007. 1.195 Seiten. 44,- Euro. ISBN 978-3-8329-1825-5. Seite $634 \mathrm{ff}$. 
Insgesamt sind 38 der Kriterien bewohnerbezogen, die übrigen beziehen sich auf die Einrichtung. Die bewohnerbezogenen Kriterien werden an einer nach dem $\mathrm{Zu}$ fallsprinzip ermittelten Stichprobe von zehn Prozent der alten Menschen untersucht. Mittels eines Punktesystems werden daraus Noten errechnet, die sich an den Schulnoten von 1 (sehr gut) bis 5 (mangelhaft) orientieren. Es gibt dabei Einzelnoten für jedes der insgesamt 64 Kriterien, die zu Teilnoten für die vier Prüfbereiche sowie einer Gesamtnote für die Einrichtung zusammengefasst werden. Zur besseren Einordnung der Heime wird dieser die Durchschnittsnote aller der bis dahin überprüften Einrichtungen auf Landesebene gegenübergestellt. rer wirtschaftlichen Existenz gefährden kann.

\section{Fehlende wissenschaftliche Grundlage}

Die Schaffung von Transparenz ist zwar prinzipiell sehr begrüßenswert, doch das vom MDK verwendete Instrument erscheint dazu völlig ungeeignet. Ein grundlegendes Problem haben die Verfasser im Vorwort der Prüfanleitung selbst beschrieben: »Die Vertragsparteien ... haben diese Vereinbarung in dem Wissen geschlossen, dass es derzeit keine pflegewissenschaftlich gesicherten Erkenntnisse über valide Indikatoren der Ergebnis- und Lebensqualität der pflegerischen Versorgung in Deutsch-

\section{"Viele Dokumentationsanforderungen werfen ethische Fragen hinsichtlich eines sgläsernen Bewohnersi auf"}

Außerdem wird eine Befragung von Bewohnern nach 18 Kriterien durchgeführt. Auch hieraus errechnet sich eine Note, die zwar ebenfalls öffentlich ausgewiesen wird, allerdings nicht in die Gesamtnote der Einrichtung einfließt. Da sich die alten Menschen in einem Abhängigkeitsverhältnis zur Einrichtung befinden und außerdem erfahrungsgemäß zu Zufriedenheitsäußerungen tendieren, wird die Aussagefähigkeit von Bewohnerbefragungen von Fachleuten generell problematisiert. Trotz dieser Einschränkung ist es aber nur schwer nachvollziehbar, dass die Sicht der Bewohner gar keinen Einfluss auf die Gesamtbewertung des Heimes hat. Die alten Menschen werden also offensichtlich nicht für fähig gehalten, ihre eigene Lebenssituation adäquat beurteilen zu können. Es zeigt sich darin ein defizitäres Bewohnerbild, welches das subjektive Erleben der alten Menschen in keiner Weise ernst nimmt.

Gemäß den Vorgaben des Pflege-Weiterentwicklungsgesetzes werden die Noten sowohl im Internet (z. B. unter www.pflegelotse.de) wie auch durch Aushang im Eingangsbereich des Heimes veröffentlicht. Dies soll zu mehr Transparenz beitragen und Heimplatzsuchende bei ihrer Entscheidung unterstützen.

Die Noten können sich für das Heim somit zu einem wichtigen Marketinginstrument entwickeln. Andererseits wird gerade in Regionen mit einem Überangebot an Heimplätzen eine "schlechte Note « für die Einrichtung vermutlich zu Nachfrageproblemen führen, die sie letztlich sogar in ih- land gibt. « Es wird also mit einem Instrument geprüft, das für die Heime die skizzierten weitreichenden Folgen haben kann, aber erklärtermaßen wissenschaftlich in keiner Weise fundiert ist.

Ein Gutachten des Pflegewissenschaftlers Prof. Dr. Stefan Görres kommt zu einem ähnlichen Ergebnis: »Die Prüfberichte ... treffen kaum Aussagen über die tatsächliche Lebenssituation pflegebedürftiger Menschen. « Dieses vernichtende Urteil bezieht sich zwar auf den vorherigen Prüfleitfaden des MDK, doch da dieser zu großen Teilen in die neue Richtlinie eingeflossen ist, kann auch die Kritik im Wesentlichen darauf übertragen werden.

In dem neuen Prüfkatalog sind im Bereich der »Pflege und medizinischen Versorgung « 33 der 35 Kriterien als » bewohnerbezogen « deklariert. Von diesen 33 angeblich bewohnerbezogenen Kriterien müssen jedoch 31 (= 93,9 Prozent) durch die Pflegedokumentation nachgewiesen werden. Dies bedeutet, dass der MDK, entgegen den Vorgaben des Gesetzgebers, nicht die Lebensqualität der Bewohner überprüft, sondern fast ausschließlich die Verschriftlichung der Leistungen. Bei einer unvollständigen Dokumentation wird, selbst wenn sich der Bewohner in einem "guten Pflegezustand « zeigt, eine mangelhafte Erbringung der Leistungen unterstellt, die sich negativ auf die Note auswirkt. Da für die Einrichtungen aber eine überdurchschnittliche Note von so großer Bedeutung ist, sind sie somit gezwungen, ihre Aktenführung weiter zu optimieren. Wie bereits zuvor ausgeführt, geht dies bei gleichbleibender Personalausstattung zulasten der praktischen Pflege.

Die Dokumentationsanforderungen des MDK sind so weitreichend, dass sie außerdem grundlegende ethische Fragen aufwerfen. Letztlich wird dabei gefordert, "gläserne Bewohner « zu schaffen, die nicht nur in ihrem aktuellen Befinden, sondern auch in ihrer Biografie jeglicher Intimsphäre beraubt werden. Dem liegt ein katastrophales Missverständnis biografischer Arbeit zugrunde, denn diese darf niemals ein Selbstzweck sein, sondern hat nur dann ihre Berechtigung, wenn daraus Konsequenzen für die aktuelle Betreuung eines Bewohners resultieren.

\section{Keine einheitliche Umsetzung}

Ein weiteres Problem zeigen die stark differierenden Ergebnisse der Prüfungen in den verschiedenen Bundesländern. Die Diskrepanz der Durchschnittsnoten zwischen Baden-Württemberg und Hamburg um 1,7 Punkte legt die Vermutung einer uneinheitlichen Umsetzung des Instruments nahe. In der Praxis ist zudem festzustellen, dass die Prüfungen auch personenabhängig sind. So ist mir eine Einrichtung bekannt, die den Bewohnern ein umfangreiches, sogar preisgekröntes Veranstaltungsprogramm bietet. Dort wird zwar die Teilnahme der Bewohner dokumentiert, nicht jedoch, wie von den Prüfern gefordert, deren Reaktionen und Befindlichkeiten während der Veranstaltungen. Auf den Einwand der Heimleitung, dass bei Umsetzung dieser Forderungen aufgrund des zeitlichen Aufwandes das Veranstaltungsangebot sicherlich reduziert werden müsse, entgegneten ihm der MDKMitarbeiter: "Dann ist das auch nicht schlimm. «Eine Aussage, die an Zynismus kaum zu überbieten sein dürfte.

In den Medien ist im Zusammenhang mit den Überprüfungen der Heime in Anspielung auf den »Technischen Überwachungsverein « häufig vom »Pflege-TÜV « die Rede. Eine Bezeichnung, welche die Realität offensichtlich genau trifft, denn es geht dabei nicht um die Lebenszufriedenheit der Bewohner, sondern um die korrekte Anwendung von Dokumentationstechniken.

\section{Gerichtliche Klagen anhängig}

Aufgrund dieser Zusammenhänge ist es nur verständlich, dass zwischenzeitlich gerichtliche Klagen gegen das Verfahren anhängig sind. Einer der Kläger ist die CaritasBetriebsführungs- und Trägergesellschaft $\mathrm{mbH}$ (CBT). Sie lässt sich vor Gericht von dem renommierten Sozialrechtler Prof. Dr. Thomas Klie vertreten, der zur Gesamtproblematik bemerkt: »Der MDK macht die 
Qualität der Pflege ganz wesentlich an den Eintragungen in der Pflegedokumentation fest - und dies mangels tragfähiger Indikatoren für die Ergebnisqualität. «CBT-Geschäftsführer Franz Stoffer führt dazu ergänzend aus, dass »der mit den Prüfungen verbundene bürokratische Aufwand nicht zu rechtfertigen (ist). Durchschnittlich wendet ein Heim mittlerer Größe 80 Stunden für die Begleitung und Nachbereitung der MDK-Prüfung auf, Zeit, die für die Begleitung und Assistenz der pflegebedürftigen Bewohner nicht zur Verfügung steht. «

Zusammenfassend lässt sich festhalten, dass die Note die Lebensqualität der Bewohner in keiner Weise abbildet. Das Instrument täuscht nicht nur die Verbraucher, die sich davon eine Hilfestellung bei der Auswahl geeigneter Anbieter erhoffen, sondern es führt vor allem zu einem weiteren Anstieg der Bürokratie in den Heimen, welche erhebliche negative Auswirkungen auf die Pflege- und Betreuungsqualität hat.

\section{Vergaberecht und Sozialrecht: Ein Widerspruch?}

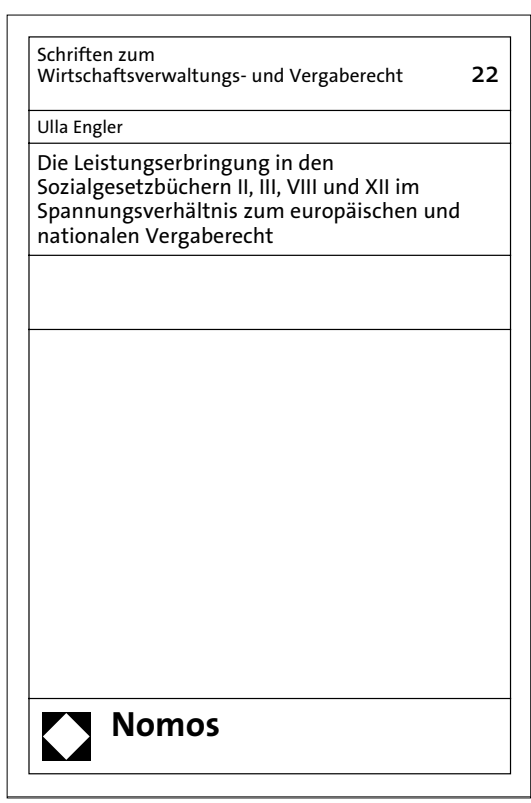

Die Leistungserbringung in den Sozialgesetzbüchern II, III, VIII und XII im Spannungsverhältnis zum europäischen und nationalen Vergaberecht Von RAin Dr. Ulla Engler 2010, 221 S., brosch., 59,-€, ISBN 978-3-8329-5315-7 (Schriften zum Wirtschaftsverwaltungs- und Vergaberecht, $B d .22)$
In Zeiten knapper öffentlicher Kassen ist das Vergaberecht auch im sozialen Bereich zunehmend von Bedeutung. Hierbei stellt sich jedoch die Frage nach der Vereinbarkeit von Sozialrecht und Vergaberecht.

Vor diesem Hintergrund stellt die Verfasserin anhand aktueller Rechtsprechung die Leistungserbringung in den Bereichen der Grundsicherung für Arbeitsuchende, der Arbeitsförderung, der Kinder- und Jugendhilfe und der Sozialhilfe dar und zeigt auf, wo die sozialrechtliche Leistungserbringung im Spannungsverhältnis zum Vergaberecht steht. Während das europäische Vergaberecht in erster Linie Rahmenbedingungen vorgibt, resultieren aus dem nationalen Vergaberecht konkrete Vorgaben, die öffentliche Auftraggeber bei der Vergabe öffentlicher Aufträge zu beachten haben. Schwerpunkt der Arbeit ist die Frage, ob bei der Leistungserbringung in den SGB II, III, VIII und XII, die vorwiegend im Rahmen eines sozialrechtlichen Dreiecksverhältnisses erbracht wird, überhaupt ein öffentlicher Auftrag im Sinne des Vergaberechts vorliegt.

Die Autorin verfügt als Rechtsanwältin bei einem Spitzenverband der Freien Wohlfahrtspflege über praktische Erfahrung aus „beiden Welten“, dem Sozialrecht und dem Vergaberecht.

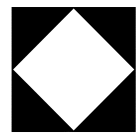

\section{Nomos}

